Proceedings of the WELCOME Scientific Meeting on Hybrid Nanostructures, Toruń, Poland, August 28-31, 2011

\title{
Interactions of Photosystem I with Plasmonic nanostructures
}

\author{
M. Hussels ${ }^{a}$, J.B. Nieder ${ }^{b, *}$, C. ElsësSer ${ }^{b}$ AND M. BreChT $^{a, \dagger}$ \\ ${ }^{a}$ Universität Tübingen, IPTC and Lisa + Center, Auf der Morgenstelle 18, 72076 Tübingen, Germany \\ ${ }^{b}$ Fachbereich Physik, Freie Universität Berlin, Arnimallee 14, 14195 Berlin, Germany
}

\begin{abstract}
The plasmonic interaction effects of various nanostructures on the fluorescence properties of photosystem I as found by single-molecule spectroscopy are summarized. The used nanostructures are spherical Au nanoparticles, silver island films as well as hexagonal arrays of nanometer-sized Au- and Ag-triangles (the Fischer patterns). The fluorescence emission of photosystem I is intensified due to coupling with these nanostructures. For single photosystem I complexes, enhancement factors of up to 37 were observed. The average enhancements vary between 2.2 for Au Fischer pattern and 9 for spherical Au nanoparticles. The enhancement of the fluorescence of photosystem I demonstrates in all cases a strong wavelength dependence. This wavelength dependence can be explained by the spatially largely extended multichromophore composition of photosystem I complexes. From the viewpoint of the usability of these nanostructures for spectroscopic signal enhancement, the Fischer patterns are beneficial, due to their very low autoluminescence.
\end{abstract}

PACS: $73.20 . \mathrm{Mf}, 87.64 . \mathrm{kv}, 87.80 . \mathrm{Nj}$

\section{Introduction}

Metallic nanostructures in combination with proteins connected to their surface (bio-nanohybrids) provide one of the most adaptable architecture for design and implementation of bio-functionality at the nanoscale $[1,2]$. The broadness of the field of applications relies on the malleable characteristics of nanostructures combined with the vast diversity of protein functions [2]. One field of research deals with improving the applicability of plasmonic nanostructures for absorption/emission enhancement of fluorophores [3]: this is useful for e.g. increasing sensitivity of fluorescence-based assays in drug discovery and high throughput screenings.

Biophysical applications using nanostructures must be carefully designed because the interaction between proteins and metal nanostructures can lead to modifications of both interaction partners. While molecular adsorbates on the surfaces of nanoparticles can modify the surface plasmon resonances [4], the plasmonic interaction can also affect protein function [5]. As a consequence, a deep understanding of the fundamental interaction mechanisms between metal nanostructures and proteins is necessary for the design of hybrids with tailored properties.

An advantage for all techniques using the absorption/ emission properties of the chromophores is that chromophores close to metal nanoparticles are less prone to optical saturation and thus have a higher maximum emission rate and show dramatically decreased fluorescence

\footnotetext{
* Present address: ICFO - Institut de Ciencies Fotoniques, Av Carl Friedrich Gauss num. 3, 08860 Castelldefels, Barcelona, Spain.

$\dagger$ corresponding author
}

lifetimes [6]. Thus nanoparticles can be utilized to enhance the signal of chromophores in a simple way, a beneficial effect especially for single-molecule techniques, where the detection of fluorophores is often limited by low signal intensities.

In this work, we compare the effects of the coupling of photosystem I (PSI) to ordered and unordered nanoparticle assemblies. The unordered assemblies are: silver island films (SIF) and colloidal solutions of spherical $\mathrm{Au}$ nanoparticles (AuNP); and the ordered nanostructures are periodic patterns of hexagonal arranged $\mathrm{Au}-(\mathrm{Au} \Delta)$ or Ag-triangles $(\operatorname{Ag} \Delta)$.

The Ag nanostructures of the SIF are of various widths and heights as can be seen in the atomic force microscopy (AFM) scan of the surface (see Fig. 1a). The colloidal gold nanospheres show a more uniform size with a diameter of $100 \mathrm{~nm}$ and are randomly distributed in the sample. The hexagonal arranged $\mathrm{Au} \Delta$ or $\mathrm{Ag} \Delta$ are also known as Fischer patterns, an AFM image of such type of structures is shown in Fig. 1b [7]. Such patterns can be produced by nanosphere lithography [8,9]; an inexpensive, inherently parallel, high-throughput nanofabrication technique capable of producing well-ordered 2D periodic nanostructure arrays from all different kinds of vaporizable materials $[8,9]$. The size of the nanostructures can be tuned by varying the basis length of the structuring nanospheres and their thickness can be controlled by the metal's evaporation conditions.

The experiments described here were carried out on spatially separated single PSI trimers. Hybrids made of PSI and metal nanoparticles serve as a potential building block of hybrid solar cells. The capability of PSI hybrids for the production of biofuels such as $\mathrm{H}_{2}$ was shown by Grimme et al. in Ref. [10]. In addition, PSI serves as a model system for biological light harvesting and charge separation processes. PSI's main function is to capture and convert solar energy into electrical energy. Almost 100 chlorophylls per PSI monomer are involved in light 

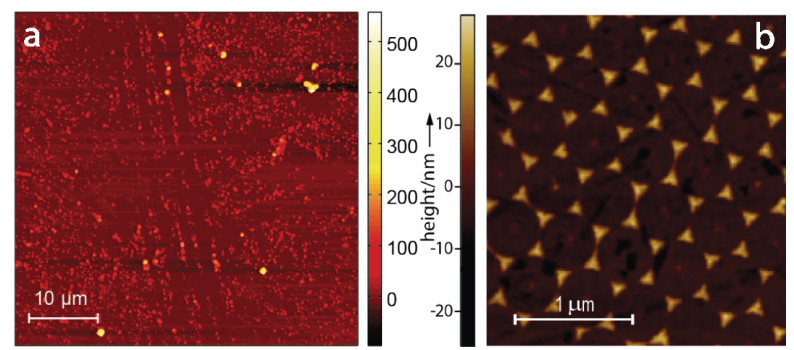

Fig. 1. AFM images of $\operatorname{SIF}$ (a) and $\mathrm{Au} \Delta$ (b) taken with modifications from Refs. [5, 32].

harvesting, excitation energy transfer and charge separation [11-13]. PSI can be regarded as a natural solar cell absorbing across a wide spectral range covering $\approx 45 \%$ of the solar radiation at Earth's surface. The unique quantum yield of $\approx 1$ for charge separation contributes to the attraction of PSI for electrochemical approaches for the conversion of light energy into electrical [14-18] or chemical energy $[10,19,20]$. Among the 100 chlorophylls some chlorophyll dimers and trimers with lower site energies than the reaction center with its main absorbance at $700 \mathrm{~nm}$ (P700) were found [21-26]. Although activation energy for the uphill energy transfer towards P700 is required, these low energy chlorophylls are nevertheless involved in the excitation energy transfer towards the reaction centers. Due to these astonishing properties, the so-called red chlorophylls are of great interest and their function is still under debate $[25,26]$. The transfer from the different red states to P700 is blocked at low temperatures and the energy is partially released as fluorescence emission detectable down to the single-molecule level [27-29]. Several of these red chlorophylls contribute to the fluorescence spectrum of single PSI trimers. They differ in their site energies, intensities and susceptibility for spectral diffusion. Therefore, sharp stable lines as well as broad intensity distributions are present in the spectra of single PSI complexes. The sharp lines represent chlorophylls that are affected by spectral diffusion with low rates. The probability of observing those sharp lines stable in wavelength is high in the wavelength region between 700 and $715 \mathrm{~nm}$. The broad intensity distributions are due to chlorophylls that are affected by spectral diffusion with much higher rates. These distributions dominate the spectra at longer wavelength [29, 30].

\section{Material and methods}

Isolated and purified PSI trimers from Th. elongatus (procedure described in Ref. [31]) were diluted in buffer solution for single-molecule measurements as described in Refs. [5, 32]. The experiments on PSI trimers are comprised of two different experiments. One experiment was performed starting with a PSI solution obtained from the resolubilization of PSI crystals [5] and the other with a PSI solution where glycerol was added for storage at cryogenic temperatures [32]. To couple PSI to AuNP (PSI-
AuNP) an excess of colloidal gold nanospheres of approximately $100 \mathrm{~nm}$ diameter (BBInternational) was added to the PSI buffer solution (AuNP:PSI ratio ca. 12:1). To couple PSI to the nanostructures (SIF, $\mathrm{Au} \Delta$ and $\mathrm{Ag} \Delta$ ) the diluted PSI solution was placed on top of these surfaces, a detailed description is given in Refs. [5, 32]. The preparation of SIF was performed as described in paper of Chowdhury et al. [33].

Figure 1a shows an AFM image of a SIF structure as used for the described experiments. The Fischer patterns were produced by nanosphere lithography as described in Refs. [8, 32].

Figure 1b shows an AFM image of an $\mathrm{Ag} \Delta$ structure as used for the described experiments. Experiments were carried out using a home-built confocal microscope operating at 1.3 to $1.4 \mathrm{~K}$ as recently described in Ref. [34]. The usual exposure time for each spectrum was $1 \mathrm{~s}$ in a sequence of spectra resulting in a typical $\mathrm{S} / \mathrm{N}$-ratio of $>6$ for single uncoupled PSI complexes at an excitation power of $100 \mu \mathrm{W}$.

\section{Results and discussion}

Figure 2 shows a collection of fluorescence intensity scans taken for the various PSI containing samples as well as for the bare nanostructures. All scans are taken under identical experimental conditions and shown with an identical intensity color scale. The scans in the first row $2 \mathrm{a}-\mathrm{e}$ are recorded on a sample of pure PSI and on the hybrid samples composed of highly diluted PSI and the respective nanoassembly, they are referred as: uncoupled PSI, PSI-AuNP, PSI-Au $\Delta$, PSI-SIF, and PSI-Ag $\Delta$ respectively [5, 32]. A qualitative increase of the intensity from the sample solely containing PSI to the PSI-hybrids is obvious. In the second row $2 \mathrm{f}-\mathrm{i}$ intensity scans taken on the bare nanostructures are shown [5,32]. The Au $\Delta$ show minor intensity contributions, barely observable with the given intensity scale (Fig. 2g). For $\mathrm{AuNP}, \mathrm{Ag} \Delta$, and SIF intense spots can be observed even without the presence of PSI complexes. The spots of $\operatorname{Ag} \Delta$ are lower in intensity than those observed in the scan of PSI- $\mathrm{Ag} \Delta$ and the contributions are uniformly distributed over the whole scanned area. The signal intensities of the SIF and AuNP are comparable with those detected for PSI containing samples, but the number of spots per sample area is comparatively small.

The spectral analysis of these luminescence contributions shows that the signals from the nanostructures are well distinguishable from PSI fluorescence emission, see Refs. [5, 32]. To study the influence of the nanostructures on the fluorescence emission properties of PSI, spectra from intense spots in the pure and in the hybrid samples were collected, sorted out if associated to nanostructure luminescence, and compared. All spectra taken on various intensity spots of the PSI-Au $\Delta$ as well on the PSI$\operatorname{Ag} \Delta$ show PSI fluorescence emission characteristics and thus sorting was not necessary for these hybrid structures. 

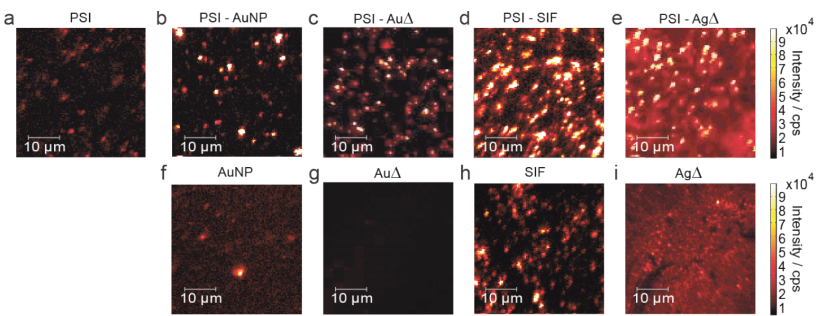

Fig. 2. Fluorescence intensity scans of PSI/PSI-nanohybrids (first row) and of the nanoassemblies without PSI (second row): scans of PSI on bare quartz (a), PSI-AuNP (b), PSI-Au $\Delta$ (c), PSI-SIF (d), and PSI$\operatorname{Ag} \Delta(\mathrm{e}), \operatorname{AuNP}(\mathrm{f}), \operatorname{Au} \Delta$ (g), $\operatorname{SIF}(\mathrm{h})$, and $\operatorname{Ag} \Delta$ (i). The fluorescence intensity scans were acquired with an integration time of $2 \mathrm{~ms}$ per pixel. An identical color-scale range was chosen for all data sets. Images are taken from Refs. [5, 32].

Overall, 162 sequences for PSI-Au $\Delta, 158$ for PSIAuNP, 118 sequences for PSI-Ag $\Delta, 72$ for PSI-SIF, and 295 for PSI in buffer were recorded. The sequences for PSI in buffer are based on two different sample preparations (see Sect. 2). For each preparation 148 and 147 sequences were taken as described in Refs. [5, 32].

The distribution of enhancement factors was determined by comparing the intensities of single PSI complexes in the respective hybrid sample to the average intensity in the uncoupled configuration. For this procedure, an average intensity for uncoupled PSI must be determined.

The collection of the intensities of uncoupled PSI complexes shows a nearly Gaussian shaped distribution as shown in the histogram in Fig. 3. The maximum of this distribution corresponds to the mean intensity of uncoupled PSI (Fig. 3) and can be equalized with an enhance- ment factor of 1 . The $x$-axis of the histogram is normalized to this mean, thus defining the enhancement factor. Figure 3 shows the distribution of enhancement factors for PSI-Au $\Delta$ and PSI-Ag $\Delta$. The appropriate distributions for PSI-AuNP and PSI-SIF can be found in Fig. 3 in Ref. [5]. The distributions for PSI-Aus (blue) and PSI-Ag $\Delta$ (lilac) — as well as for PSI AuNP and PSISIF [5] - are asymmetric, largely extending to the high-intensity sides. The maximum intensity found for an individual PSI-Au $\Delta / \mathrm{PSI}-\mathrm{Ag} \Delta$ measures $10.5 / 15$ times the average intensity of uncoupled PSI. The average enhancement factor based on the histograms is 2.2 for PSI$\mathrm{Au} \Delta$ and 5.7 for PSI-Au $\Delta$. For PSI-SIF and PSI-AuNP, the maximum observed enhancements are 36 and 37 ; and the average enhancements are 7 and 9 , respectively. The different enhancement values are collected in Table.

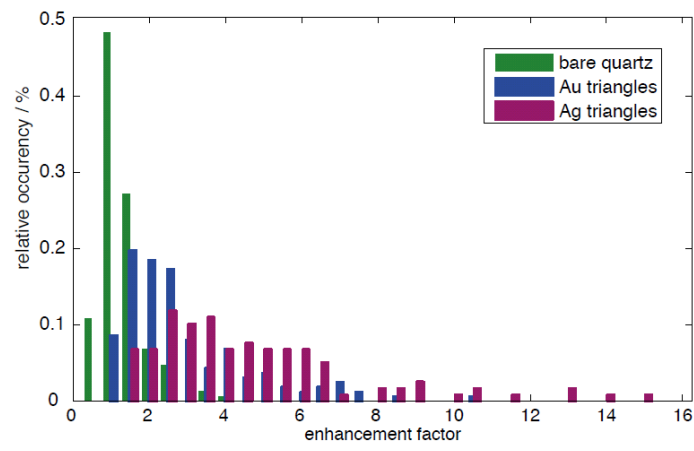

Fig. 3. Intensity histograms of PSI signals (green), $\mathrm{PSI}-\mathrm{Au} \Delta$ (blue), and PSI-Ag $\Delta$ (lilac). The intensities of the individual complexes were obtained from spectrally resolved data. The counts detected in the wavelength region $695-780 \mathrm{~nm}$ were integrated after subtraction of a constant background to eliminate dark counts and stray light. Finally, these values were summarized for the first $120 \mathrm{~s}$ of the sequences. The figure is taken with modifications from Ref. [32].

\section{TABLE}

The deviations* of the maximum values are due to differences in the PSI preparation, see Sect. 2 and Refs. [5, 32].

\begin{tabular}{l|c|c|c|c|c}
\hline \hline \multicolumn{1}{c|}{ Sample } & PSI & PSI-Au $\Delta$ & PSI-AuNP & PSI-Ag $\Delta$ & PSI-SIF \\
\hline emission maximum & $729.9 / 727^{\star}$ & 729.5 & 732 & 731.3 & 719 \\
FWHM & $27.6 / 33^{*}$ & 33.3 & 52 & 38.7 & 37 \\
average enhancement & - & 2.2 & 9 & 5.7 & 7 \\
max. enhancement & - & 10.5 & 37 & 15 & 36
\end{tabular}

Plasmonic interaction generally depends on the spectral resonance condition between chromophores and surface plasmons, their intersystem distance and their relative orientations. The electric field close to the nanostructures shows a strong spatial variation $[35,36]$. For triangles in hexagonal Fischer patterns very high field enhancements are observed at positions on the tips (apex) of the triangles, whereas at the edges of vanishing field en- hancements are found [35, 36]. Anger et al. [37] observed the following distance dependence for fluorescence emission enhancement for a fluorophore in resonant coupling conditions to a spherical AuNP: quenching of the fluorescence at distances shorter than approximately $2 \mathrm{~nm}$, maximum enhancement at approximately $5 \mathrm{~nm}$, and exponential decay at longer distances leading to vanishing enhancements at around $80 \mathrm{~nm}$ distance. In the investi- 
gated samples, the PSI complexes are randomly oriented with respect to the plasmonic structures. As a consequence, PSI complexes will be present in regions with both low as well as high field enhancement, resulting in the broad distribution of enhancement factors observed (Fig. 3). The enhancement factors observed for PSISIF and PSI-AuNP are comparable with the reported values for other fluorophore-metal nanostructure assemblies, whereas the enhancement factors for PSI-Au $\Delta$ and PSI-Ag $\Delta$ are smaller in magnitude [5, 6, 37-42].

All plasmonic nanostructures lead to increased emission of the deposited PSI complexes. The evaluation of data for PSI-SIF and PSI-AuNP is more demanding, because in these cases the background signals of the nanostructures are not negligible. An analysis to distinguish between contributions stemming from PSI and that from the nanostructures proved unnecessary for PSI-Au $\Delta$ and PSI-Ag $\Delta$. This provides an advantage as compared to the strong background signals observed for spherical AuNP and SIFs [5]. Although, the arrays of $\mathrm{Au} \Delta$ and $\operatorname{Ag} \Delta$ show smaller enhancement values as compared to AuNP and SIF, the low autoluminescence makes these nanostructures preferential tools for spectroscopy.

Figure 4 shows the average spectra of all spectra taken on individual uncoupled PSI, PSI-AuNP, PSI-SIF, PSI$\operatorname{Ag} \Delta$, and PSI-Au $\Delta$. The spectra were scaled to similar magnitude. The maxima positions and the full width at half maximum (FWHM) are given in Table. Modifications of the emission profiles induced by interaction with the nanostructures are clearly visible. These modifications depend on the wavelength e.g. the emission at the blue side of the profile shows much stronger enhancement than that found in the red part of the spectrum.

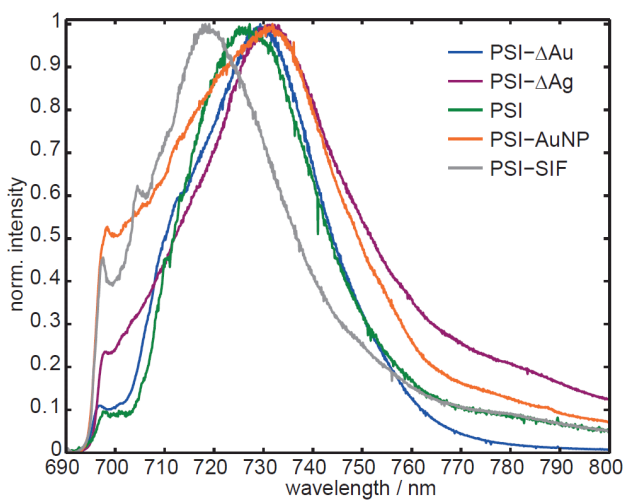

Fig. 4. Averaged spectra obtained from summation of spectra from all single PSI complexes (from Th. elongatus) obtained for uncoupled PSI, PSI-AuNP, PSI-SIF, PSI-Au $\Delta$, and PSI-Ag $\Delta$. The spectra were scaled to similar maximum values.

The wavelength-dependence of the enhancement can be obtained by dividing the average spectra of the different hybrids by that of uncoupled PSI. The resulting curves for the enhancement are shown in Fig. 5. It should be noted that the procedure chosen here depends cru-

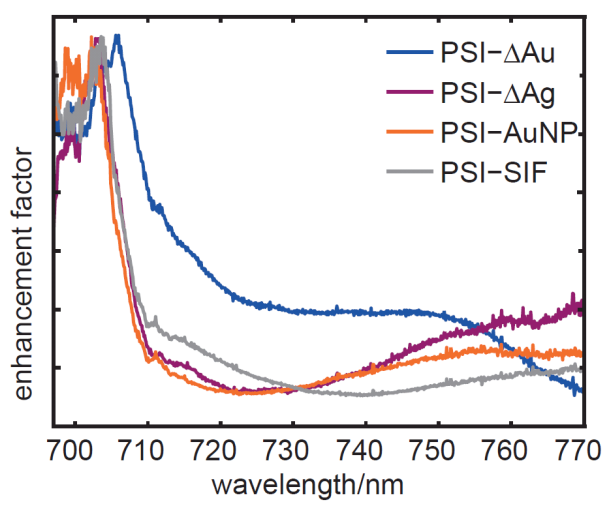

Fig. 5. Wavelength dependence of the fluorescence enhancement. For this representation the average spectra of PSI-AuNP, PSI-SIF, PSI-Au $\Delta$, and PSI-Ag $\Delta$ were divided by the average spectrum of uncoupled PSI. The data were scaled to similar amplitude.

cially on the elimination of background contributions and consequently the error for the determination of the enhancement factor becomes larger in the spectral region with low intensity. For better comparability the curves are scaled to similar maximum/minimum values in the given wavelength range. The fluorescence emission in the range 698-712 nm shows large relative enhancements for all structures. Compared to that, the wavelength region $>712 \mathrm{~nm}$ remains rather featureless.

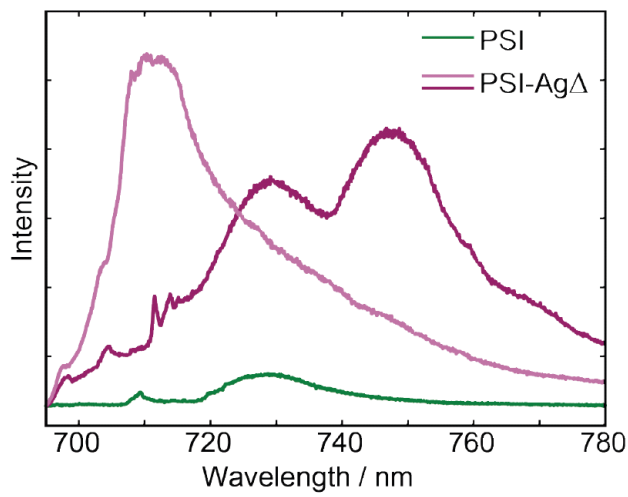

Fig. 6. Three spectra of single PSI complexes taken with identical experimental settings are shown: uncoupled PSI (green) and PSI-Ag $\Delta$ (lilac). Taken with modifications from Refs. [32].

Figure 6 shows the emission spectra of one uncoupled PSI complex (green line) and two single PSI complexes coupled to $\operatorname{Ag} \Delta$ ( light and dark lilac). The spectra of these PSI-Ag $\Delta$ represent extreme cases of wavelength-dependent enhancement. One spectrum (light lilac) is highly enhanced in the region around $710 \mathrm{~nm}$. Compared with the average value of uncoupled PSI, this region is enhanced by a factor of $\approx 100$. The strong enhancement of the short-wavelength region indicates an enhanced fluorescence deactivation of the higher energy antenna pig- 
ments, which show virtually no fluorescence in absence of plasmonic structures. The other spectrum (black) shows an example for strong enhancement at the red edge of the PSI emission. The emission in the red spectral region is due to the emission of far-red chlorophylls. The fluorescence of these chlorophylls is completely quenched if the reaction center is in the oxidized form $\left(\mathrm{P}_{000}{ }^{+}\right)$. The oxidized form is in almost all cases present in the uncoupled PSI, because the high excitation rates used during the experiments are much higher than the rate of charge recombination [26]. Under these experimental conditions the emission of the far red states is quenched by $\mathrm{P} 700^{+}$ and escapes the detection in uncoupled PSI [26]. The high intensity of this contribution can only be explained by a dramatic change of the time scales involved in the quenching processes, e.g. the fluorescence lifetime of the far-red chlorophylls, energy transfer to the quenching site or charge recombination $\left(\mathrm{P} 700^{+} \rightarrow \mathrm{P} 700\right)$.

The shape of the emission spectra of the PSI-nanoparticle hybrids shows clear deviations from linear enhancement. Such deviations stand in contrast to observations for single chromophores and two-chromophore FRET-coupled systems as the peridinin-chlorophyll protein close to plasmonic structures $[3,6]$.

The extinction spectra of the used nanostructures show only a weak variation $(\approx 20 \%)[2,36,43]$ over the emission range of PSI. This variation is much smaller than the variation of the enhancement factor observed for the PSI-nanohybrids (Fig. 5). Therefore we assume that the observed emission profile changes cannot be explained by simple convolution of PSI emission with the plasmon spectra (plasmonic shaping). The deviations from the linear enhancement must rather be rationalized by intrinsic properties of PSI, as they are: a large lateral extension of the molecular complex and the specific couplings of the chromophores within the complex.

The shape of a PSI trimer can be approximated by a cylinder measuring around $20 \mathrm{~nm}$ in diameter and approximately $5 \mathrm{~nm}$ in height.

Figure 7 shows a scheme where the extension of PSI is compared to a enhancement curve as measured for spherical AuNP in resonant coupling conditions with a fluorophore according to Ref. [37]. If the PSI complex is located close to the surface of the nanoparticle, some chlorophylls are in the quenching regime, whereas others are in conditions for maximum enhancement.

Therefore, the shape of the emission spectrum of an individual PSI complexes close to a nanostructure can deviate remarkably from the shape of uncoupled PSI (Fig. 6). The chromophores in light harvesting systems are coupled to ensure fast and efficient energy transfer to the reaction centers [21]. The energy transfer efficiency depends on the spectral overlap, spatial separation, and orientation of the involved chromophores [44]. The specific coupling conditions between the chromophores lead to a characteristic set of transition rates and thus to preferred energy transfer pathways $[44,45]$. The interaction between coupled chromophores and plasmonic struc-

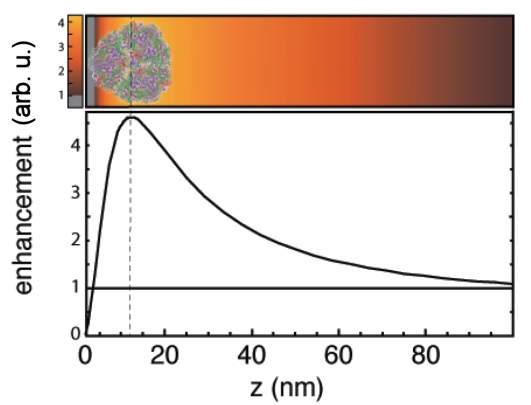

Fig. 7. Illustration of the distance dependence of the enhancement factor. On top, the enhancement factor depends on the distance between chromophores in PSI and the surface of the metal nanoparticle. The structure of a PSI trimers is given and the region of quenching and enhancement are color coded as given. The curve given below visualizes the slope of the enhancement in more detail; the curve is adapted from Ref. [37].

tures alters Förster-interaction distances between chromophores [46]. The exciton distribution on the chromophores of PSI most probably gets largely changed as a result of these plasmonic interaction effects. Additional energy transfer pathways will be formed and chlorophylls that do not participate in the native/uncoupled state could be involved. In addition, chromophores, which were almost non-fluorescent before become fluorescent. Particularly, we observed such an enhanced fluorescence emission from energetically higher lying chlorophyll states, indicating an overall reduced excitation feeding of the reaction center.

\section{Conclusion}

In conclusion, the investigated PSI-nanohybrids (AuNP, Au $\Delta$, SIF, and $\mathrm{Ag} \Delta$ ) show increased fluorescence compared to uncoupled PSI. The average enhancement varies between 2.2 and 9 . For all PSI-nanohybrids the enhancement depends strongly on the wavelength. The alteration of the shapes of the fluorescence spectra upon plasmonic interaction of PSI with the nanostructures can be explained by the spatial extension of the chromophore system in PSI together with the coupling between the chromophores. Especially, we observe additional spectral contributions on the short wavelength side of the fluorescence emission spectrum of PSI, indicating fluorescence emission from energetically higher lying states, which are almost non-fluorescent in uncoupled PSI.

\section{Acknowledgments}

Single-molecule experiments were carried out in the laboratory of Prof. Robert Bittl (Free University Berlin); his continuous support is gratefully acknowledged. Nanostructureswere prepared in the laboratory of Prof. Paul Fumagalli (Free University Berlin); his continuous support is gratefully acknowledged. The AFM images were taken in the group of Prof. J.I. Pascual and Prof. S. Reich, their support is gratefully acknowledged. We 
thank Eberhard Schlodder for PSI samples and fruitful discussions. This work is supported by Free University Berlin initiative — "Nanoscale: Nanoskalige Funktionsmaterialien", the Cluster of Excellence: "Unifying Concepts in Catalysis" and the Heisenberg Program of the DFG (BR4102/1-1, BR4102/2-1).

\section{References}

[1] K. Aslan, I. Gryczynski, J. Malicka, E. Matveeva, J.R. Lakowicz, C.D. Geddes, Curr. Opin. Biotechnol. 16, 55 (2008).

[2] J.N. Anker, W.P. Hall, O. Lyandres, N.C. Shah, J. Zhao, R.P. Van Duyne, Nature Mater. 7, 442 (2008).

[3] J.R. Lakowicz, K. Ray, M. Chowdhury, H. Szmacinski, Y. Fu, J. Zhang, K. Nowaczyk, Analyst 133, 1308 (2008).

[4] L. Shang, Y.Z. Wang, J.G. Jiang, S.J. Dong, Langmuir 23, 2714 (2007)

[5] J.B. Nieder, R. Bittl, M. Brecht, Angew. Chem.-Int. Ed. 49, 10217 (2010).

[6] S. Mackowski, S. Wormke, A.J. Maier, T.H.P. Brotosudarmo, H. Harutyunyan, A. Hartschuh, A.O. Govorov, H. Scheer, C. Bräuchle, Nano Lett. 8, 558 (2008).

[7] U.C. Fischer, H.P. Zingsheim, J. Vac. Sci. Technol. 19, 881 (1981)

[8] A. Kosiorek, W. Kandulski, P. Chudzinski, K. Kempa, M. Giersig, Nano Lett. 4, 1359 (2004).

[9] J.C. Hulteen, R.P. van Duyne, J. Vac. Sci. Technol. A-Vac. Surf. Films 13, 1553 (1995).

[10] R.A. Grimme, C.E. Lubner, D.A. Bryant, J.H. Golbeck, J. Am. Chem. Soc. 130, 6308 (2008).

[11] P. Fromme, P. Jordan, N. Krauss, Biochim. Biophys. Acta Bioenerg. 1507, 5 (2001).

[12] P. Jordan, P. Fromme, H.T. Witt, O. Klukas, W. Saenger, N. Krauss, Nature 411, 909 (2001).

[13] Photosystem I: The Light-Driven Plastocyanin:Ferredoxin Oxidoreductase, Ed. J.H. Golbeck, Springer, Berlin 2006.

[14] A.O. Govorov, I. Carmeli, Nano Lett. 7, 620 (2007).

[15] R. Das, P.J. Kiley, M. Segal, J. Norville, A.A. Yu, L.Y. Wang, S.A. Trammell, L.E. Reddick, R. Kumar, F. Stellacci, N. Lebedev, J. Schnur, B.D. Bruce, S.G. Zhang, M. Baldo, Nano Lett. 4, 1079 (2004)

[16] L. Frolov, Y. Rosenwaks, C. Carmeli, I. Carmeli, Adv. Mater. 17, 2434 (2005).

[17] A.O. Govorov, Adv. Mater. 20, 4330 (2008).

[18] N. Terasaki, N. Yamamoto, T. Hiraga, Y. Yamanoi, T. Yonezawa, H. Nishihara, T. Ohmori, M. Sakai, M. Fujii, A. Tohri, M. Iwai, Y. Inoue, S. Yoneyama, M. Minakata, I. Enami, Angew. Chem.-Int. Ed. 48, 1585 (2009).

[19] C.E. Lubner, R. Grimme, D.A. Bryant, J.H. Golbeck, Biochemistry 49, 404 (2010).

[20] H. Krassen, A. Schwarze, B. Friedrich, K. Ataka, O. Lenz, J. Heberle, ACS Nano 3, 4055 (2009).

[21] B. Gobets, R. van Grondelle, Biochim. Biophys. Acta 1507, 80 (2001).
[22] M. Byrdin, I. Rimke, E. Schlodder, D. Stehlik, T.A. Roelofs, Biophys. J. 79, 992 (2000).

[23] L.O. Palsson, J.P. Dekker, E. Schlodder, R. Monshouwer, R. van Grondelle, Photosynth. Res. 48, 239 (1996).

[24] A.N. Melkozernov, Photosynth. Res. 70, 129 (2001).

[25] N.V. Karapetyan, E. Schlodder, R. van Grondelle, J.P. Dekker, in: Advances in Photosynthesis and Respiration, vol. 24, Photosystem I: The Light-Driven Plastocyanin:Ferredoxin Oxidoreductase, Ed. J.H. Golbeck, Springer, Berlin 2006.

[26] E. Schlodder, M. Hussels, M. Cetin, N.V. Karapertyan, M. Brecht, BBA - Bioenergetics 1807, 1423 (2011).

[27] F. Jelezko, C. Tietz, U. Gerken, J. Wrachtrup, R. Bittl, J. Phys. Chem. B 104, 8093 (2000).

[28] A.F. Elli, F. Jelezko, C. Tietz, H. Studier, M. Brecht, R. Bittl, J. Wrachtrup, Biochemistry 45, 1454 (2006).

[29] M. Brecht, H. Studier, A.F. Elli, F. Jelezko, R. Bittl, Biochemistry 46, 799 (2007).

[30] M. Brecht, Mol. Phys. 107, 1955 (2009).

[31] P. Fromme, H.T. Witt, Biochim. Biophys. Acta Bioenerg. 1365, 175 (1998).

[32] M. Brecht, M. Hussels, J.B. Nieder, C. Elsässer, Chem. Phys., http://dx.doi.org/10.1016/j.chemphys.2012.05.005 (2012).

[33] M.H. Chowdhury, K. Ray, K. Aslan, J.R. Lakowicz, C.D. Geddes, J. Phys. Chem. C 111, 18856 (2007).

[34] M. Hussels, M. Brecht, Biochemistry 50, 3628 (2011).

[35] B.C. Galarreta, P.R. Norton, F. Lagugne-Labarthet, J. Phys. Chem. C 114, 19952 (2010).

[36] B.C. Galarreta, E. Harte, N. Marquestaut, P.R. Norton, F. Lagugne-Labarthet, Phys. Chem. Chem. Phys. 12, 6810 (2010).

[37] P. Anger, P. Bharadwaj, L. Novotny, Phys. Rev. Lett 96, 113002 (2006).

[38] Y. Chen, K. Munechika, D.S. Ginger, Nano Lett. 7, 690 (2007).

[39] S. Kuhn, U. Hakanson, L. Rogobete, V. Sandoghdar, Phys. Rev. Lett. 97, 017402 (2006).

[40] J. Enderlein, Biophys. J. 78, 2151 (2000).

[41] P.P. Pompa, L. Martiradonna, A. Della Torre, F. Della Sala, L. Manna, M. De Vittorio, F. Calabi, R. Cingolani, R. Rinaldi, Nature Nanotechnol. 1, 126 (2006).

[42] S. Vukovic, S. Corni, B. Mennucci, J. Phys. Chem. C 113, 121 (2009).

[43] J.R. Lakowicz, Y.B. Shen, S. D'Auria, J. Malicka, J.Y. Fang, Z. Gryczynski, I. Gryczynski, Anal. Biochem. 301, 261 (2002).

[44] M. Byrdin, P. Jordan, N. Krauss, P. Fromme, D. Stehlik, E. Schlodder, Biophys. J. 83, 433 (2002).

[45] M. Brecht, V. Radics, J.B. Nieder, R. Bittl, Proc. Natl. Acad. Sci. USA 106, 11857 (2009).

[46] J. Zhang, Y. Fu, M.H. Chowdhury, J.R. Lakowicz, J. Phys. Chem. C 111, 11784 (2007). 